BIO Web of Conferences 4, 00002 (2015)

DOI: $10.1051 /$ bioconf/20150400002

(C) Owned by the authors, published by EDP Sciences, 2015

\title{
Chemical evolution and life
}

\author{
Christophe Malaterre ${ }^{\mathrm{a}}$ \\ Département de philosophie, Chaire de recherche UQAM en philosophie des sciences, Université du \\ Québec à Montréal, 455 Boulevard René-Lévesque Est, Case postale 8888, succursale Centre-Ville, \\ Montréal (Québec) H3C 3P8, Canada
}

\begin{abstract}
In research on the origins of life, the concept of "chemical evolution" aims at explaining the transition from non-living matter to living matter. There is however strong disagreement when it comes to defining this concept more precisely, and in particular with reference to a chemical form of Darwinian evolution: for some, chemical evolution is nothing but Darwinian evolution applied to chemical systems before life appeared; yet, for others, it is the type of evolution that happened before natural selection took place, the latter being the birthmark of living systems. In this contribution, I review the arguments defended by each side and show how both views presuppose a dichotomous definition of "life".
\end{abstract}

\section{Introduction}

Since the formation of the Earth some four billions years ago, the chemical state of the planet has changed, leading to the appearance of living organisms, and it continues to do so. Generally understood in the context of research on the origins of life, the concept of "chemical evolution" accounts for such change. In addition to being descriptive, it also ambitions to have an explanatory force, that of explaining the very emergence of life on Earth and possibly elsewhere in the universe, by identifying the underlying processes of this emergence. In this respect, the concept of "chemical evolution" has tight links with the concept of Darwinian evolution and is often defined in reference to natural selection. This is precisely what Melvin Calvin does when he defines chemical evolution as a transposition of "evolution by natural selection" to the prebiotic world of inanimate matter [1]. For others however [2, 3], chemical evolution cannot be defined in such a way. Rather, chemical evolution is what happens before any "evolution by natural selection" might take place, such evolution by natural selection being the birthmark of living systems. This disagreement about the role of natural selection is at the core of deep disagreements about what chemical evolution stands for and what its processes could consist of. In this contribution, I first describe the context of origins of life studies (Sect. 2) and of the usage of the concept of "chemical evolution" (Sect. 3). I then analyze the Darwinian view of chemical evolution as proposed by Calvin (Sect. 4) and I contrast this view with the non-Darwinian view of Joyce and de Duve (Sect. 5). Based on this comparison, I show that both accounts rely on a dichotomous definition of "life" according to which

\footnotetext{
${ }^{\text {a }}$ Corresponding author: malaterre.christophe@uqam.ca
}

This is an Open Access article distributed under the terms of the Creative Commons Attribution License 4.0, which permits unrestricted use, distribution, and reproduction in any medium, provided the original work is properly cited. 
something is either alive or not-alive. In turn (Sect. 6), I discuss how relaxing this presupposition by adopting a more gradual definition of life can alleviate the tension between these two extreme construals of chemical evolution.

\section{Historical overview of chemical evolution}

Nobel prize laureate Melvin Calvin is often said to have fathered the notion of "chemical evolution" [4]. Calvin's view about chemical evolution is well developed in his 1969 book Chemical Evolution, but it goes back, at least, to a series of articles and reports in the 1950s in which Calvin proposes to define chemical evolution as an evolutionary process somehow similar to, and in continuity with, Darwinian evolution, but transposed to the purely chemical world that preceded the appearance of life on primitive Earth some four billion years ago $[1,5]^{1}$. It is also in these articles and reports that Calvin makes ample use of catalysts - his main research focus - to try and illustrate how such a chemical evolution could have worked.

As is often the case with concepts in general, the idea of such a chemical evolution can be found earlier in the literature. For instance, already in the 1930s, one of the early proponents of research on the origins of life, the Russian biochemist Alexander Oparin wrote about the topic and claimed that "the simplest living organisms originated gradually by a long evolutionary process of organic substances" $[9$, p. 60]. Also, in the 1910s, in a book about the origin and the nature of life, the British chemist Benjamin Moore referred, in quite lyrical terms, to a "process of chemical evolution" that "brought life to the womb of our ancient mother earth in the far distant Palaeozoic ages" [10, p. 191] $]^{2}$. Earlier still, claims of a "chemical evolution" could be found in the writings of the French biologist Félix Le Dantec, a disciple of Pasteur [11] $]^{3}$. And a few decades in his Principles of Biology, Herbert Spencer mentioned the idea of an "inorganic evolution" and of an "evolution of organic matter" that would have preceded the evolution of living forms [13, pp. 480-488].

Along the same lines of thought, Calvin proposed to construe "chemical evolution" as "the time for the formation of chemicals of various degrees of complexity upon the surface of the Earth, but before the appearance of systems that we could call living" [1, p. 4]. And this conception of chemical evolution is the one that is often referred to in his later writings [e.g. 5]. So defined, the notion of "chemical evolution" is purely descriptive and corresponds to a time period during which a natural phenomenon occurred: that by which some of the simple chemical compounds that were present on primitive Earth came to react and interact with one another, and led to the appearance of the very first living systems, somehow between the formation of the Earth 4.5 billion years ago [e.g. 14], and the earliest most likely traces of life 3.5 billion years ago $[15,16]$.

\section{Revisiting chemical evolution}

This broad descriptive view of chemical evolution as proposed by Calvin is generally well accepted by proponents of the non-Darwinian view as well. Nevertheless, a closer look shows that such a construal of chemical evolution is somehow too narrow on at least three aspects.

First, it is generally accepted that there is no reason to limit chemical evolution to the surface of the Earth, nor to the type of chemistry that takes place on Earth. Chemical evolution most certainly

\footnotetext{
1 The term "chemical evolution" is also encountered in astronomy to refer to the synthesis and distribution of chemical elements in particular regions of the cosmos [e.g. 6, 7] and in evolutionary biology with the meaning of "molecular evolution" [e.g. 8]. In this contribution, I limit my analysis to the context of research on the origins of life.

${ }^{2}$ I thank Alan Schwartz for kindly bringing this reference to my attention.

${ }^{3}$ Quoted in [12, p. 101].
} 


\section{ORIGINS}

took place in the primitive atmosphere of Earth, its clouds and storms [e.g. 17] as well as way deep in oceans around hydrothermal vents [e.g. 18], but also in many regions of the universe, not just Earth: interstellar space, meteorites, exoplanets and many other astronomical bodies. In fact, complex organic molecules have been found on meteorites, including for instance lipid-like molecules and amino-acids [e.g. 19-21], or even in the interstellar medium, including more than a hundred organic compounds that may serve as starting points for a complex interstellar chemistry [e.g. 22].

Second, defining chemical evolution as a process that only concerns chemical molecules or types of chemical molecules, may impose too restrictive limitations if one also wishes to bridge the gap to biological evolution. In particular, supra-molecular systems ought to be taken into account: such supra-molecular systems consists of sets of molecules that are often structurally organized -and sometimes functionally- and that are quite likely to be found on the way to life. For instance, amphiphile molecules form micelles and vesicles when in aqueous solution under specific conditions [e.g. 23]. Other organic molecules can form cross-catalytic networks, be they nucleic acids or oligopeptides for instance [e.g. 24, 25]. The formation of structural assemblies and functional sets of very diverse types of molecular compounds ought, therefore, to also be accounted for in chemical evolution.

Third, whereas Calvin chose to limit chemical evolution to the period that precedes the appearance of living systems, no such limitation is actually necessary: why would chemical evolution stop at once as soon as living systems appear? On the opposite, chemical evolution is likely to continue somehow in parallel with biological evolution, even if under different forms or differing degrees. It is also most certainly still taking place in many areas in the universe.

As a result, the perimeter of chemical evolution initially defined by Calvin deserves to be somehow enlarged, even if the part of chemical evolution that is relevant to explaining the appearance of living systems on Earth is of very special interest to us. More broadly construed, chemical evolution therefore corresponds to the formation of chemicals and chemical systems of various degrees of complexity, in the universe, or in subsets of the universe such as Earth, and in particular, but not exclusively, before the presence of living entities.

Such a definition, captures what many, if not most, scientists working on the origins of life now have in mind when referring to chemical evolution [e.g. 26-30]. Yet, if such a definition clarifies the scope of the natural phenomenon that one wishes to label as chemical evolution, it does not provide any explanation of this phenomenon by means of specific evolutionary processes or mechanisms. Calvin proposes to look to evolutionary biology for insights.

\section{Chemical evolution according to Calvin}

What Calvin makes explicit in the 1950s is the central explanatory role that a biologically-inspired evolutionary process, namely that of natural selection, could play within chemical evolution. For Calvin, the very mechanism of chemical evolution is nothing but the mechanism of natural selection taken in its simplest form as the interplay of variation, reproduction and selection. The theory of chemical evolution therefore appears as an immediate extension of natural selection to an abiotic world of molecules.

Following his early interests in catalysis, and just after the publication of Miller's work on the abiotic synthesis of amino acids [17], Calvin envisions a prebiotic evolution of more and more sophisticated catalysts as one of the major contributions to the processes that could bridge non-living matter and living matter. With the Darwinian paradigm in mind, Calvin sets clear objectives for the concept of chemical evolution: "the term "evolution" [...] calls for the possibility of random variation amongst systems together with a mechanism for selecting amongst those random variations [...]. What I would like to do is to extend the very same terms into non-biological systems and show that they apply" [1, pp. 3-4]. The interplay of variation, reproduction and selection is a central theme of Calvin's 
notion of chemical evolution. He proposes that prebiotic chemical reactions may have led to the random synthesis of various organic compounds [17, 31], thereby generating, by accumulation, a broad molecular diversity upon which some selective pressures could have been exerted. For Calvin, the "essence of the process of selection" lies among the properties of these chemical compounds, and most specifically with the property of autocatalysis: compounds that can catalyze, even only slightly, their own formation, will outnumber those that cannot. And the random synthesis, via prebiotic chemistry, of ever more efficient autocatalysts is thought to have fueled the early evolution of chemical systems. Yet, to make chemical evolution similar to Darwinian evolution, Calvin also envisions a hypothetical process of cumulative improvement by which specific autocatalysts could be able to evolve by randomly acquiring novel substructures or by slightly modifying their composition or conformation. Calvin lacks direct experimental support concerning the existence of such autocatalysts but grounds his proposal on an analogy with ever more complex families of known catalysts, such as those based on aqueous ferric ions [1, p. 12].

It is the overall interplay of variation, replication and selection among prebiotic chemical compounds that results in chemical evolution. For Calvin, both processes of chemical evolution and of biological evolution remain the same: they are based on Darwinian selection. Yet, if the processes are the same, they apply to different entities. And it is the nature of these entities that delineate chemical evolution from biological evolution: non-living chemical entities on the one hand, living entities on the other. The appearance of life therefore serves as the major, if not sole, dividing line between chemical evolution and biological evolution.

\section{The non-Darwinian view of chemical evolution}

A more recent usage of the concept of chemical evolution has seen a shift from Calvin's Darwinian view to a non-Darwinian view. According to this non-Darwinian view, chemical evolution consists of evolutionary processes that are totally different from natural selection, the latter being understood to be the true birthmark of biological evolution. Gerald Joyce for instance, a prominent researcher in the field of origins of life, defends such a view when he claims that "the worlds of prebiotic chemistry and primitive biology lie on opposite sides of the defining moment for life, when Darwinian evolution first began to operate [...]. Once a general mechanism existed for self-replication, allowing the introduction of variation and the ability to replicate those variants, Darwinian evolution began to operate. This marked the beginning of life" [2, pp. 214-215]. By setting apart prebiotic chemistry and Darwinian evolution, each on one side of the origin of life event, Joyce reduces chemical evolution to prebiotic chemistry, that is to say to a set of chemical reactions that would have happened spontaneously on primitive Earth, within the limits of what is thermodynamically and kinetically possible. Of course, processes of selection were possible under chemical evolution: such processes are typically associated with faster chemical reactions. Processes of variation would also fit with this view of chemical evolution provided they are understood as sets of chemical reactions resulting in the spontaneous accumulation of random varied compounds. Yet any interplay between these processes, and in particular the association of replication to variation and selection so as to result in some sort of selection for cumulative variation would result in a shift from chemical evolution to Darwinian biological evolution.

In such a non-Darwinian view of chemical evolution, also espoused for instance by de Duve [3], the dividing line between chemical evolution and biological evolution consists, at the same time, in a shift in the nature of the evolutionary process and in a change in the nature of the evolving entities. The process of Darwinian selection clearly belongs to biological evolution and delineates biological evolution from chemical evolution, the latter being more or less reduced to prebiotic chemistry. And at the same time, like in the Darwinian view, the very nature of the evolving entities changes from non-living to living as one goes from chemical evolution to biological evolution. 


\section{ORIGINS}

\section{Life as demarcation criteria?}

Both the Darwinian and the non-Darwinian accounts of chemical evolution use "life" as a key criteria to distinguish chemical evolution from biological evolution. Yet both views rest on, what I will argue is, a faulty premiss according to which it is possible to clearly delineate living from non-living matter. I propose four reasons for rejecting "life" as a demarcation criteria.

First, there is a wide disagreement about defining life. Definitions of life abound [e.g. 32] and yet, there is still no agreement on any of them: counter-examples are often offered that defy even the most elaborate definitions. Border-line entities such as viruses are often invoked, some arguing that they should not be included within the circle of living systems in so far as they lack metabolic activity [e.g. 33, 34], others arguing the contrary, for instance grounding their arguments on the viral factories that virus generate [e.g. 35]. Some also argue that self-replicating strands of RNA, like those of the RNA world scenario [e.g. 27] should qualify as living [e.g. 33] whereas others disagree for lack of metabolic activity and membrane enclosure [e.g. 36, 37]. And there are many other border-line cases including autocatalytic networks [e.g. 38] and protocells [e.g. 28, 39]. As such, the lack of a generally accepted definition of life makes it problematic to use "life" as demarcation criteria.

This disagreement about defining life shows that there is a fairly wide range of ways of delineating living matter from non-living matter. Incidentally, this also points to a "gray-zone" in between what one would intuitively qualify as living and what one would definitely count as non-living. One can therefore argue that there is no sharp delineation between non-living and living matter, and that "life", as we know it today on Earth, can somehow be considered as the outcome of a succession of "more-or-less alive" systems. As a result, any dichotomous definition of life is bound to fail. Instead, life ought to be defined as a fuzzy or multi-valued predicate based on a zero-to-one scale [40, 41]. The ranking of any "more-or-less alive" system along this scale would then result from a sum of its different life-like functions: for instance, a system may be capable of metabolic activity without being able to reproduce itself; another system may be able to self-replicate without any metabolic activity; yet another one may be capable of metabolism and of reproduction, yet not of variation; and so forth. In addition, any such system may be more-or-less successful along any of these functional dimensions, for instance at making copies of itself, at generating variants of itself, at harnessing energy and metabolites etc. As a result, one can conceive the "gray-zone" in between non-living and living matter as being populated by a broad range of systems, each being "more-or-less alive" in its own way [42]. The existence of such a multidimensional gray-zone helps reconcile differing viewpoints about definitions of life and borderline cases; yet it also argues against the possibility to use "life" as a clear cut demarcation criteria that could be used to delineate biological evolution from chemical evolution.

Thirdly, if one wishes anyway to adopt a dichotomous definition of life -with a view to using it as demarcation criteria- one will soon face a dilemma with respect to excluding from evolutionary biology some of the entities that are studied within this discipline. Indeed, dichotomous definitions of life tend to exclude entities such as viruses and plasmids from the set of living systems. If one adopts such a strict definition of life as demarcation criteria of biological evolution, then the evolution of such entities should not be explained by biological evolution but by chemical evolution. Yet this is clearly not the case, as viruses and plasmids are usually considered as part of the entities that do evolve by natural selection [e.g. 43].

Lastly, taking life as demarcation criteria also brings with it the risk of defining chemical evolution as a set of teleological processes leading to life. Life - as we know it-certainly is an outcome of chemical evolution. Yet defining chemical evolution on the basis of this outcome is problematic and carries a heavy teleological connotation. Furthermore, this may also tend to exclude chemical evolutionary phenomena that may not lead to life but yet be interesting in themselves, or that may lead to other forms of life (than the one we know on Earth). 
Once the requirement for a dichotomous definition of life is relaxed and a more gradual definition accepted, the tension between the Darwinian and the non-Darwinian view of chemical evolution disappears. The reason is twofold. On the one hand, the Darwinian view no longer requires the clear distinction between non-living matter and living matter at their point of junction, which makes chemical evolution gradually flow into biological evolution. On the other, a gradual construal of life makes it impossible to choose at the same natural selection as the birthmark of life. As a consequence, natural selection can also apply to chemical systems that are not necessarily fully alive, thereby becoming a possible evolutionary process of chemical evolution.

\section{Conclusion}

While Calvin proposes to construe chemical evolution in a purely Darwinian sense, others argue in favor of a non-Darwinian account that restricts evolution by natural selection to biological evolution. Such perspectives appear at first glance irreconcilable. However, both rest on the assumption that a dichotomous construal of "life" can be used to part living entities from non-living ones. If a more gradual construal of "life" is used instead, the tension between the Darwinian view and the non-Darwinian view of chemical evolution dissolves. And I have argued that are indeed good reasons to adopt such a gradual view of life. Furthermore, these reasons are also good reasons to consider chemical evolution and biological evolution on a continuum. Whereas paradigm cases of biological evolution are clear cases of natural selection, other cases of biological evolution either rely on evolutionary processes that are different from natural selection -for instance drift or epigenetic mechanisms- or deal with entities that are not clearly alive -such as viruses or plasmids. Furthermore, research on the origins of life indicates the existence of a "gray-zone" of systems that may be more-or-less alive along different dimensions. Whereas Calvin [1] like Joyce [2] and de Duve [3] tend to consider chemical evolution and biological evolution as making a point-to-point connection, this connection being defined by a sharp transition either in terms of entities or in terms of processes articulated on the basis of a strictly dichotomous concept of "life', there are also good -and likely better- reasons to conceive the transition from chemical evolution to biological evolution as gradual along multiple connections both in terms of evolutionary processes and evolving entities. Such an evolutionary continuum may, in turn, also help conceive of chemical evolution as a much more elaborate and rich framework than is usually thought of.

Earlier versions of this paper were presented at the 2009 ISHPSSB meeting in Brisbane and the 2010 PSA meeting in Montréal, and I wish to thank the audiences for very helpful comments. The paper benefited from collaborative interactions stimulated by CNRS interdisciplinary program "Environnements planétaires et origines de la vie" and COST Action TD 1308. Part of this research was conducted with support from UQAM research chair in philosophy of science.

\section{References}

[1] Calvin, M. (1955) "Chemical Evolution and the Origin of Life"

[2] Joyce, G.F. (2002) The antiquity of RNA-based evolution. Nature 418 (6894), 214-221

[3] De Duve, C. (2005) The onset of selection. Nature 433, 581-582

[4] Ferris, J.P., and Hagan Jr, W.J. (1984) HCN and chemical evolution: the possible role of cyano compounds in prebiotic synthesis. Tetrahedron 40 (7), 1093-1120

[5] Calvin, M. (1969) Chemical Evolution: Molecular Evolution Towards the Origin of Living Systems on the Earth and Elsewhere, Clarendon P

[6] Edvardsson, B., Andersen, J., Gustafsson, B., et al. (1993) The chemical evolution of the galactic disk-part one-analysis and results. Astron. Astrophys. 275, 101

[7] Pei, Y.C., and Fall, S.M. (1995) Cosmic chemical evolution. Astrophys. J. 454, 69 


\section{ORIGINS}

[8] Gillespie, J.H. (1991) The causes of molecular evolution, Oxford University Press

[9] Oparin, A.I. (1953) Origin of Life, Dover Pubns, New York

[10] Moore, B. (1913) The origin and nature of life, H. Holt and Company

[11] Le Dantec, F.A. (1896) Théorie nouvelle de la vie, F. Alcan, Paris

[12] Cerceau, F.R., and Bilodeau, B. (2009) Les origines de la vie: histoire des idées, Ellipses

[13] Spencer, H. (1864) The Principles of Biology, Williams and Norgate

[14] Despois, D., and Gargaud, M. (2006) A Synthetic Interdisciplinary "Chronological Frieze": an Attempt. Earth Moon Planets 98 (1-4), 291-297

[15] Schopf, J.W. (2006) Fossil evidence of Archaean life. Philos. Trans. R. Soc. B Biol. Sci. 361 (1470), 869-885

[16] Brasier, M., McLoughlin, N., Green, O., and Wacey, D. (2006) A fresh look at the fossil evidence for early Archaean cellular life. Philos. Trans. R. Soc. B Biol. Sci. 361 (1470), 887-902

[17] Miller, S.L. (1953) A production of amino acids under possible primitive earth conditions. Science 117 (3046), 528-529

[18] Huber, C., and Wächtershäuser, G. (1998) Peptides by activation of amino acids with CO on (Ni, Fe) S surfaces: implications for the origin of life. Science 281 (5377), 670-672

[19] Cooper, G., Kimmich, N., Belisle, W., et al. (2001) Carbonaceous meteorites as a source of sugarrelated organic compounds for the early Earth. Nature 414 (6866), 879-883

[20] Deamer, D.W. (1985) Boundary structures are formed by organic components of the Murchison carbonaceous chondrite. Nature 317, 792-794

[21] Glavin, D.., Matrajt, G., and Bada, J.. (2004) Re-examination of amino acids in Antarctic micrometeorites. Adv. Space Res. 33 (1), 106-113

[22] Guillemin, J.-C., Bouyahyi, M., and Hassan Riague, E. (2004) Prebiotic, planetary and interstellar chemistry starting from compounds detected in the interstellar medium. Adv. Space Res. 33 (1), $81-87$

[23] Monnard, P.-A., and Deamer, D.W. (2002) Membrane self-assembly processes: Steps toward the first cellular life. Anat. Rec. 268 (3), 196-207

[24] Sievers, D., and Von Kiedrowski, G. (1994) Self-replication of complementary nucleotide-based oligomers. Nature 369 (6477), 221-224

[25] Ashkenasy, G., Jagasia, R., Yadav, M., and Ghadiri, M.R. (2004) Design of a directed molecular network. Proc. Natl. Acad. Sci. U. S. A. 101 (30), 10872-10877

[26] Eigen, M., and Schuster, P. (1977) A principle of natural self-organization. Naturwissenschaften 64 (11), 541-565

[27] Gilbert, W. (1986) Origin of life: The RNA world. Nature 319 (6055)

[28] Szostak, J.W., Bartel, D.P., and Luisi, P.L. (2001) Synthesizing life. Nature 409 (6818), 387-390

[29] Leslie E., O. (2004) Prebiotic Chemistry and the Origin of the RNA World. Crit. Rev. Biochem. Mol. Biol. 39 (2), 99-123

[30] Eschenmoser, A. (2007) The search for the chemistry of life's origin. Tetrahedron 63 (52), $12821-12844$

[31] Garrison, W.M., Morrison, D.C., Hamilton, J.G., et al. (1951) Reduction of Carbon Dioxide in Aqueous Solutions by Ionizing Radiation. Science 114 (2964), 416-418

[32] Popa, R. (2004) Between necessity and probability: searching for the definition and origin of life, Springer

[33] Luisi, P.L. (1998) About various definitions of life. Orig. Life Evol. Biosph. 28 (4-6), 613-622

[34] Ruiz-Mirazo, K., Peretó, J., and Moreno, A. (2004) A universal definition of life: autonomy and open-ended evolution. Orig. Life Evol. Biosph. 34 (3), 323-346

[35] Raoult, D., and Forterre, P. (2008) Redefining viruses: lessons from Mimivirus. Nat. Rev. Microbiol. 6 (4), 315-319

[36] Shapiro, R. (1986) Origins: A Skeptic's Guide to the Origin of Life on Earth, Summit Books 
[37] Segré, D., Ben-Eli, D., Deamer, D.W., and Lancet, D. (2001) The lipid world. Orig. Life Evol. Biosph. 31 (1-2), 119-145

[38] Kauffman, S.A. (1993) The origins of order: Self-organization and selection in evolution, Oxford university press

[39] Noireaux, V., Bar-Ziv, R., Godefroy, J., et al. (2005) Toward an artificial cell based on gene expression in vesicles. Phys. Biol. 2 (3), P1-P8

[40] Bruylants, G., Bartik, K., and Reisse, J. (2010) Is it Useful to Have a Clear-cut Definition of Life? On the Use of Fuzzy Logic in Prebiotic Chemistry. Orig. Life Evol. Biospheres 40 (2), 137-143

[41] Bedau, M.A. (2010) An Aristotelian Account of Minimal Chemical Life. Astrobiology 10 (10), 1011-1020

[42] Malaterre, C. (2010) Lifeness signatures and the roots of the tree of life. Biol. Philos. 25 (4), 643-658.

[43] Godfrey-Smith, P. (2009) Darwinian populations and natural selection, Oxford University Press 